\title{
Dúvidas de pacientes sobre condutas de profissionais de saúde durante a internação
}

\section{hospitalar}

\author{
Patients 'questions about the conducts of health professionals during hospital intervention \\ Preguntas de los pacientes sobre el comportamiento de los profesionales sanitarios durante la \\ hospitalización
}

Recebido: 05/06/2021 | Revisado: 11/06/2021 | Aceito: 12/06/2021 | Publicado: 04/07/2021

Cristina Berger Fadel

ORCID: https://orcid.org/0000-0002-7303-5429

Universidade Estadual de Ponta Grossa, Brasil

E-mail: cbfadel@gmail.com

Melina Lopes Lima

ORCID: https://orcid.org/0000-0002-8266-4040

Universidade Estadual de Ponta Grossa, Brasil

E-mail: enfmelina@gmail.com

Letícia Gomes Cordeiro

ORCID: https://orcid.org/0000-0003-0778-5085

Universidade Estadual de Ponta Grossa, Brasil

E-mail: legommmes@gmail.com

Alessandra de Souza Martins

ORCID: https://orcid.org/0000-0001-6345-8412

Universidade Estadual de Ponta Grossa, Brasil E-mail: alessandraphn@hotmail.com

Luciane Patrícia Andreani Cabral

ORCID: https://orcid.org/0000-0001-9424-7431

Universidade Estadual de Ponta Grossa, Brasil E-mail: luciane.pacabral@gmail.com

Camila Zanesco

ORCID: https://orcid.org/0000-0001-8326-0390

Universidade Estadual de Ponta Grossa, Brasil E-mail: camila_zanesco@hotmail.com

\begin{abstract}
Resumo
O objetivo deste estudo é apreender dúvidas de pacientes sobre condutas de saúde realizadas por diferentes profissionais durante o período de internação hospitalar. Trata-se de um estudo qualitativo descritivo, realizado com 63 pacientes de um hospital universitário, que afirmaram ter apresentado dúvida sobre o tratamento realizado pela equipe de saúde durante a internação hospitalar. Os dados foram obtidos por meio de entrevista telefônica gravada realizada ao próprio paciente ou destinada a um membro familiar ou cuidador. Os resultados foram analisados e expressos em duas categorias e quatro subcategorias. A primeira categoria, mais prevalente, traz as incertezas quanto aos tratamentos ou procedimentos que foram realizados durante a internação hospitalar. A segunda categoria considerou as dúvidas quanto a diagnósticos médicos não conclusivos e quanto à falta de consenso entre os diferentes profissionais da saúde. A falha da comunicação entre profissional e paciente ficou evidenciada nos relatos dos sujeitos deste estudo. Esse resultado reforça a importância de investimentos em relações interpessoais, comunicação e literacia tanto na formação de profissionais quanto na prática dos serviços de saúde.

Palavras-chave: Pacientes; Incerteza; Internação hospitalar; Relações profissional-paciente; Equipe de assistência ao paciente.

Abstract

The aim of this study is to apprehend patients' doubts about health behaviors performed by different professionals during the hospitalization period. This is a descriptive qualitative study, carried out with 63 patients from a university hospital, who said they had doubts about the treatment performed by the health team during hospitalization. The data were obtained through a recorded telephone interview conducted with the patient or with a family member or caregiver. The results were analyzed and expressed in two categories and four subcategories. The first category, more prevalent, brings uncertainties regarding the treatments or procedures that were performed during hospitalization. The second category considered doubts about non-conclusive medical diagnoses and the lack of consensus among different health professionals. The failure of communication between professional and patient was evidenced in the reports of the subjects of this study. This result reinforces the importance of investments in interpersonal relationships, communication and literacy both in the training of professionals and in the practice of health services.
\end{abstract}


Keywords: Patients; Uncertainty; Hospitalization; Professional-patient relations; Patient care team.

\section{Resumen}

El objetivo de este estudio es captar las dudas de los pacientes sobre las conductas de salud realizadas por diferentes profesionales durante la estancia hospitalaria. Se trata de un estudio cualitativo descriptivo, realizado con 63 pacientes de un hospital universitario, quienes manifestaron tener dudas sobre el tratamiento realizado por el equipo de salud durante su estancia hospitalaria. Los datos se obtuvieron a través de una entrevista telefónica grabada realizada con el paciente o dirigida a un familiar o cuidador. Los resultados se analizaron y expresaron en dos categorías y cuatro subcategorías. La primera categoría, más prevalente, trae incertidumbres sobre los tratamientos o procedimientos que se realizaron durante la hospitalización. La segunda categoría consideró las dudas sobre diagnósticos médicos no concluyentes y la falta de consenso entre los diferentes profesionales de la salud. El fracaso de la comunicación entre el profesional y el paciente se evidenció en los informes de los sujetos de este estudio. Este resultado refuerza la importancia de las inversiones en las relaciones interpersonales, la comunicación y la alfabetización tanto en la formación de los profesionales como en la práctica de los servicios de salud.

Palabras clave: Pacientes; Incertidumbre; Internación hospitalaria; Relaciones profesional-paciente; Equipo de asistencia al paciente.

\section{Introdução}

A habilidade humana de comunicar-se com outros seres da sua mesma espécie é uma das ferramentas mais importantes para o adequado convívio social, sendo a comunicação advinda de aspectos verbais a forma mais relevante dentre as interlocuções no âmbito da saúde (Warrillow; Farley; Jones, 2016).

No contexto da abordagem da comunicação em saúde entre profissionais, pacientes, cuidadores e familiares,inúmeros são os entraves e desafios enfrentados. Todo o processo sofre interferência de questões relacionadas aos próprios indivíduos e envolvidos, aspectos sociais, culturais, emocionais e psicológicos, fatores atrelados ao campo profissional (no caso de trabalhadores) e vinculados à presença ou ausência de experiências com os serviços de saúde (no caso de usuários), concorrem diretamente para a relação interativa entre os indivíduos (Warrillow; Farley; Jones, 2016).

Especificamente diante do processo de hospitalização, o paciente e o familiar acabam passando por situações estressantes, desgastantes e de fragilidade, as quais podem potencializar o sentimento de incapacidade (Warrillow; Farley; Jones, 2016). Esse impacto emocional da internação hospitalar pode, então, desencadear o surgimento de dúvidas sobre o tratamento prestado. Para a psicologia, familiares encontram frequentemente dificuldades em acompanhar seu ente durante o processo de internamento, sendo elas: o afastamento do emprego, de seu domicílio e muitas vezes a necessidade de deixar de cuidar de seu familiar hospitalizado para prestar assistência a outro (Warrillow; Farley; Jones, 2016). Fragilizados, familiares ainda recebem diagnósticos e prognósticos que muitas vezes não são bem compreendidos e exemplificados gerando-se, a partir disso, uma espécie de crise relacional entre os sujeitos e as equipes de saúde (Warrillow; Farley; Jones, 2016).

Nesse viés, segundo a Política Nacional de Humanização (PNH), editada em 2013, a comunicação é uma das diretrizes do processo de humanização da assistência, ou seja, é direito do paciente expressar seus sentimentos permitindo que a equipe de saúde o retorne de maneira profissional e humana em auxilio de suas necessidades (Brasil a, 2013). A comunicação eficaz está, cada vez mais sendo reconhecida como uma habilidade não técnica fundamental para todos os profissionais de saúde (Warrillow; Farley; Jones, 2016). Compreende-se que a comunicação eficiente, de forma direta, confere significado e transmite confiabilidade, facilitando o processo de aderência ao tratamento e, de forma indireta, além de, contribuir para um atendimento humanizado, seja na prevenção, no processo de cura, na reabilitação ou na promoção da saúde.

No entanto, ainda que a PNH se constitua avanço para mudanças nos modos de gerir e cuidar a saúde pública no Brasil, estudos demonstram que parte dos estabelecimentos prestadores de serviços, incluindo profissionais de saúde, ainda não conseguem seguir integralmente os preceitos dessa política (Brasil a, 2013). Desta forma, apesar da preocupação com a humanização no ambiente hospitalar ser objeto atual de reflexão e discussão entre profissionais e a sociedade científica, a falha 
ou quebra da comunicação entre profissional e paciente acarretam em desentendimentos, pseudoinformações e dúvidas sobre a doença e o tratamento, com consequente geração de problemas advindos (Koerich; Erdmann; Lanzoni,2020).

Levando em consideração o processo adscrito e a importância da exploração da interface entre a escuta e a fala no campo da saúde, este estudo tem como objetivo apreender dúvidas de pacientes sobre condutas de saúde realizadas por diferentes profissionais durante o período de internação hospitalar.

\section{Metodologia}

Trata-se de um estudo de natureza qualitativa e descritiva (Gil, 2008), de abordagem dedutiva, o qual faz parte de um projeto de maior abrangência, realizado com dados primários de 514 pacientes que estiveram internados no período de fevereiro a julho de 2018, em um Hospital Universitário, considerando-se a Unidade de Terapia Intensiva (UTI) e as clínicas: cirúrgica, médica, infectologia e neurologia.

Os dados foram obtidos por meio de entrevista telefônica para o próprio paciente, ou a um membro familiar ou cuidador, valendo-se de questionário semiestruturado. Seis pesquisadores foram previamente treinados para realização da coleta de informações, além disso, foi realizado estudo piloto para avaliação do emprego dos instrumentos e calibração dos envolvidos.

Para participação na pesquisa, foram considerados os pacientes com 18 ou mais anos, que permaneceram internados no hospital por período superior a um dia; membro familiar ou cuidador que tenha acompanhado integralmente o processo de internamento (quando o próprio indivíduo não apresentava condições de responder ao questionário) e ter recebido alta hospitalar, no mínimo, 30 dias antes da realização da entrevista. Foram excluídos da amostra: gestantes; pacientes com vírus da imunodeficiência humana e hepatites virais; pacientes que tiveram procedimento cirúrgico cancelado e aqueles que permaneceram internados no Pronto Atendimento.

Os sujeitos foram previamente informados sobre os objetivos da pesquisa, seu caráter de voluntariedade e de nãoidentificação, assim como sobre a forma de coleta, análise e destino dos dados. Os que aquiesceram com a participação, o fizeram mediante o preenchimento de um Termo de Consentimento Livre e Esclarecido.

Os dados utilizados neste recorte, foram provenientes das respostas angariadas por meio da questão disparadora: "Durante a sua internação, o (a) senhor(a) ficou com alguma dúvida sobre o tratamento que foi realizado pela equipe de saúde do hospital?"

As gravações referentes a essa questão foram transcritas na íntegra por um único pesquisador. A totalidade de respostas dos sujeitos que apresentaram alguma dúvida ou insegurança sobre as condutas de saúde durante a internação (n=63) foram consideradas para análise.

O método da análise de conteúdo proposto por Bardin (2016), foi utilizado para o tratamento e exame do material gerado pelas entrevistas, com a utilização de procedimentos sistemáticos e objetivos de descrição do conteúdo das mensagens. O tema central previamente definido perpassou por três diferentes fases: pré-análise, exploração do material e tratamento dos resultados até interpretação e inferência. As narrativas que não se encaixavam no escopo desta análise não foram consideradas para o presente estudo. As categorias e subcategorias encontradas, estão expostas no quadro 01, com frequências absolutas e relativas, considerando-se o número total de ocasiões em que cada ideia foi citada. As narrativas consideradas mais representativas foram descritas.

Para aprofundar a análise, esta pesquisa se ancorou na Política Nacional de Humanização (Brasil a, 2013) e na Política Nacional de Atenção Hospitalar (Brasil b, 2013).

O estudo foi aprovado por um Comitê de Ética em Pesquisa envolvendo Seres Humanos, respeitando os ditames da resolução 466/12 do Conselho Nacional de Saúde, e pela direção técnica do referido hospital de ensino. Para garantir o 
anonimato, os participantes da pesquisa não foram identificados.

\section{Resultados e Discussão}

Do total de entrevistas tabuladas, $87,7 \%(n=451)$ dos sujeitos investigados responderam não possuir dúvidas sobre as condutas dos profissionais de saúde durante o seu período de internação hospitalar e 12,3\% (n=63) afirmaram ter apresentado alguma dúvida, sendo então considerados para o estudo.

Os sujeitos incluídos, eram em sua maioria homens $54 \%$ (n=34), casados ou com união estável 55,5\% (n=35), com

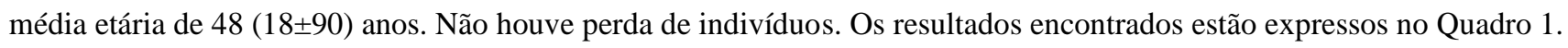

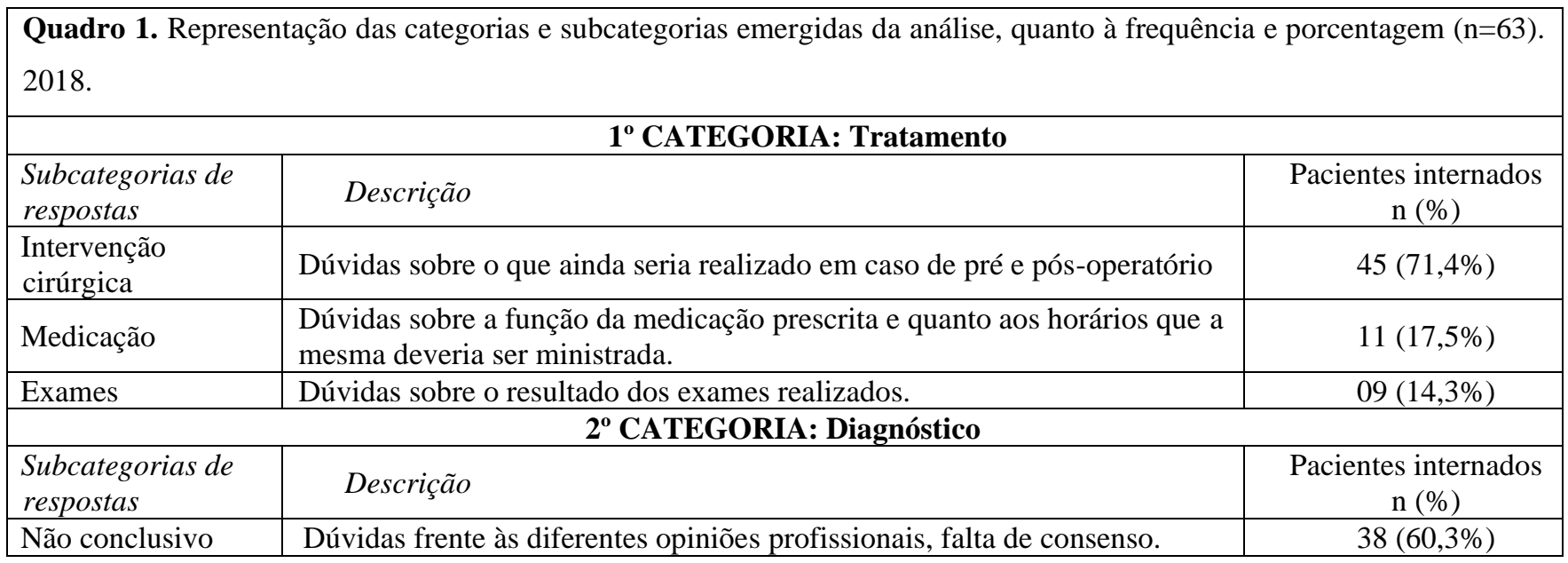

Fonte: Dados da pesquisa.

Com base no Quadro 1, é possível perceber a representatividade de cada categoria e subcategoria emergida, se destaca que a categoria tratamento, obteve maior expressão pelos participantes.

As adversidades na relação médico-paciente constituem-se objeto de discussão na área da saúde, frequentemente abordadas desde as décadas de 60 e 70 (Caprara; Franco, 1999). Nesse sentido, a formação hegemônica dos profissionais, intensamente orientada por aspectos biológicos e clínicos, é evidenciada historicamente como questão que contribui negativamente para a melhoria da qualidade e redução de gastos nos serviços de saúde (Caprara; Franco, 1999). Essa tese, permanece pertinente nos dias atuais, e demonstra os impactos negativos na assistência à saúde e na comunicação do tratamento do paciente em virtude da não consideração de sua história pessoal, na sua totalidade sócio-psíquica e cultural (Barboza et al., 2020).

Especificamente em relação a formação de profissionais médicos, Coleman et al. (2016) reforçam que o ensino sobre princípios e práticas de literacia em saúde, durante a faculdade de medicina, não pode ser realizado em um único momento, devendo se apresentar sob um formato longitudinal ou integrado ao longo da formação para o alcance de resultados positivos na melhora da comunicação dos futuros profissionais com pacientes.

Levando em consideração o modelo biomédico e mecanicista do processo saúde-doença ainda muito presente na assistência à saúde, em especial no âmbito hospitalar, a negação da dimensão subjetiva do paciente e de suas singularidades se apresenta como importante desafio para a integralidade da saúde. De um lado a comunicação do tratamento do paciente de forma técnica, a partir do ideário biológico do profissional de saúde, e do outro a perda da historicidade do sujeito internado, concorrem então, para o distanciamento da obtenção do melhor resultado esperado (Otani et al., 2018; Koerich; Erdmann; Lanzoni, 2020). 
Assim sendo, o forte embasamento da ciência médica, essencialmente organicista e predominante no loco hospitalar surgem falhas de comunicação que posteriormente poderão acarretar em diversos impasses no processo de internação. Posto isto, a primeira categoria evidenciada neste estudo traz as narrativas dos pacientes e seus familiares sobre suas incertezas quanto aos tratamentos ou procedimentos que foram realizados durante a internação hospitalar, expressando o aspecto mais prevalente pesquisado $(71,4 \%)$.

Coube aqui, de forma mais expressiva, o surgimento de dúvidas em relação a procedimentos médicos executados no período de pré e pós-operatório.

"Falaram que a cirurgia dela seria feita por vídeo, mas não foi e não explicaram o porquê. Não me falaram porque não deu certo e mudaram de ideia..." (Familiar, 18 anos).

"Não explicaram sobre o pino no joelho que coloquei. Depois demorei para entender que eu não podia pisar no chão." (Paciente, 28 anos).

A PNH traz os conceitos da indissociabilidade entre gestão e atenção, do protagonismo, corresponsabilidade, autonomia dos sujeitos e da transversalização dos saberes como elementos concretos indispensáveis no processo de humanização na saúde, em contraposição ao sentido das falas dos internados pesquisados no presente estudo (Brasil a, 2013).

Embora muitos usuários demonstrem sentirem-se coagidos ao questionar sobre seu estado de saúde, no Brasil todos estão respaldados legalmente pelo direito de receber informações apropriadas nos atendimentos e expressar se compreendeu as informações e orientações recebidas desde o momento de internação até a alta e, caso ainda tenha dúvidas, solicitar esclarecimento sobre elas (Brasil c, 2013).

A comunicação terapêutica, considerada como a habilidade profissional de ajudar as pessoas a enfrentarem os seus problemas, a relacionarem-se com outros, a ajustarem-se ao que não pode ser mudado e a enfrentarem os bloqueios que impedem a funcionalidade e autonomia, tem sido empregada como estratégia promissora de melhorias nos resultados de saúde e qualidade de vida e no desempenho do exercício profissional (Koerich; Erdmann; Lanzoni, 2020).

Além de envolver aspectos psicológicos e pessoais, a falha e a falta de comunicação, a forma de fornecer o diagnóstico e a linguagem utilizada são situações que acarretam complicações no processo de saúde-doença (Koerich; Erdmann; Lanzoni, 2020; Oliveira; Zago; Thorne, 2017). A segunda fala exemplificada mostra como a falta da comunicação entre profissional e paciente pode impedir o exercício do regime de tratamento prescrito, postergando ou complicando o sucesso do tratamento.

A relação entre a adesão ao tratamento e as práticas de humanização em saúde, com empatia e comunicação efetiva está consolidada na literatura (Koerich; Erdmann; Lanzoni, 2020), mostrando ainda a influência desses fatores na facilitação da revelação diagnóstica e do enfrentamento da doença (Grosseman; Patricío, 2020; Koerich; Erdmann; Lanzoni,2020).

A complexidade da atenção ao paciente internado exige a atuação conjunta da equipe de saúde, valorizando a comunicação nas suas distintas formas, de maneira constante entre os envolvidos, incentivando as relações interpessoais profícuas e um plano de cuidado individualizado, com foco na autonomia e segurança do paciente (Koerich; Erdmann; Lanzoni,2020).

Ainda nesta categoria, foram evidenciadas dúvidas de pacientes ou familiares sobre a necessidade de utilização de medicamentos múltiplos durante a internação hospitalar e seus horários de administração. Alguns relatos evidenciaram a perspectiva do sentimento de "cobaia clínica", ao referenciarem que a medicação era alterada ou suspendida pelos médicos sem esclarecimentos prévios. 
"As minhas dúvidas eram sobre alguns medicamentos dele e os horários que as enfermeiras iam no quarto. Acho que deveria ser explicado para que serve aquele tanto de remédio e se precisa mesmo." (Familiar, 28 anos).

Segundo o Protocolo de Segurança na Prescrição, uso e Administração de Medicamentos, coordenado pelo Ministério da Saúde e Agência Nacional de Vigilância Sanitária em 2013, a informação ao paciente sobre qual medicação ele estará recebendo faz parte do procedimento operacional padrão para uma prestação de serviço de qualidade (Brasil b, 2013). A ausência de práticas seguras e de orientação quanto ao uso de medicamentos em estabelecimentos de saúde pode gerar eventos adversos evitáveis, como erros de medicação e dúvidas sobre administração de drogas, os quais tendem a impactar na rede terapêutica e nos custos em saúde (Pena; Melleiro, 2018).

Nesse contexto, ressalta-se a importância da equipe de enfermagem, desde a interpretação da prescrição médica até a avaliação dos efeitos colaterais da medicação sobre o paciente, desenvolvendo também grande função na prevenção de erro de medicações, informando os sujeitos (pacientes e familiares) durante todo o processo, garantindo assim, os preceitos éticos da profissão e o direito do paciente (Cancino et al., 2020).

Seguindo com o exposto na categoria em pauta, foram evidenciadas dúvidas sobre o resultado e interpretação de exames laboratoriais e de imagem realizados durante a internação hospitalar, como demonstra a fala a seguir.

"Sobre a cirurgia, o médico não falou que eu tinha nódulos... mas eu fiz a ressonância no hospital. Só fiquei sabendo depois, quando minha filha viu o resultado do exame." (Paciente, 47 anos).

É possível relacionar o ocorrido há duas hipóteses. A primeira se relaciona à linguagem extremamente formal e técnica utilizada pela medicina, a qual pode causar erros de interpretação para pacientes leigos; e a segunda remete a falhas na humanização do processo de trabalho em saúde (Luiz; Caregnato; Costa, 2017). Em relação ao distanciamento hierárquico de profissionais médicos e ao tecnicismo, autores que estudam a formação profissional mostram que existe ainda um sentimento de exaltação desta profissão, colocando-os em um lugar de alto nível (Grosseman; Patricío, 2020), o que pode gerar barreiras na comunicação com pacientes (Luker et al., 2017). Considerando a perspectiva da humanização no contexto hospitalar, qualquer ponto da rede pública de saúde deve assumir uma postura capaz de acolher, escutar e dar respostas adequadas aos usuários, ou seja, prestar um atendimento com resolutividade e responsabilização, orientando o paciente e a família (Koerich; Erdmann; Lanzoni, 2020; Luiz; Caregnato; Costa, 2017; PNH, 2013). Indiscutivelmente, é necessário que sejam consideradas as discussões sobre as relações humanas no processo de formação acadêmica na área da saúde, buscando a promoção de um ambiente acolhedor e seguro, tanto ao paciente quanto aos familiares (Luker et al., 2017).

Prosseguindo, a segunda categoria do estudo, menos prevalente, considerou as dúvidas de pacientes e familiares quanto a diagnósticos médicos não conclusivos e quanto à falta de consenso entre os diferentes profissionais da saúde.

"No começo não sabiam o que era, não sabiam o diagnóstico. Ainda tenho muitas dúvidas, porque não sabem ao certo o que tenho, ou não querem me falar." (Paciente, 65 anos).

"Tive muita dúvida sobre meu diagnóstico, cada hora falavam que tinha uma doença. [...] Não ficou esclarecido o motivo da crise convulsiva." (Paciente, 31 anos.)

Os resultados alcançados nesta categoria reforçam a necessidade de melhora das habilidades de comunicação entre profissionais de saúde e pacientes, uma vez que constitui dimensão central do cuidado (Luiz; Caregnato; Costa, 2017). Especificamente em situação de doenças que solicitem internação, como é o caso do presente estudo, sentimentos de dúvidas e incertezas podem dificultar a confiança e o vínculo do paciente e família com os profissionais de saúde e impactar negativamente na adesão ao tratamento (Luiz; Caregnato; Costa, 2017). 
Por meio da comunicação efetiva, valendo-se da utilização de linguagem acessível e compreensível para os diversos públicos, profissionais de saúde podem promover a pacientes e familiares a compreensão de seus problemas, meios para o seu enfrentamento e a percepção do seu papel como agente no processo do cuidado, impactando positivamente no processo saúdedoença, bem como, na qualidade e segurança do cuidado prestado (Tavakoly et al., 2020; Luiz; Caregnato; Costa, 2017).

Assim, por ser fator essencial na comunicação entre profissionais e pacientes, a literacia em saúde torna-se fundamental para o sucesso do tratamento e, consequentemente, para a qualidade de vida de indivíduos sob cuidados de saúde em ambiente hospitalar. Compreendida como a capacidade de obter, processar e interpretar informações básicas em saúde para tomar decisões adequadas, a literacia torna-se essencial para a construção da autonomia, do empoderamento dos sujeitos em seu cuidado à saúde e para a adesão ao tratamento (Sousa et al., 2020). Complementarmente, o sucesso das ações precede a participação e envolvimento das diversas instâncias e sujeitos desde o planejamento até a execução, sendo assim, há que se refletir sobre a responsabilidade de instituições hospitalares e a necessidade de treinamentos permanentes sobre literacia e habilidades de comunicação em saúde junto aos representantes.

Outro aspecto a ser aqui explorado refere-se à fragmentação no cuidado hospitalar. Apesar da temática da formação em saúde na perspectiva da interprofissionalidade ser pauta constante das agendas de saúde pública brasileiras, na prática, estudos mostram que ainda não houve o esgotamento das perspectivas uniprofissionais (Costa; Azevedo; Vilar, 2019). Vários são os fatores que se relacionam a essa questão, envolvendo a inexistência de um plano longitudinal de cuidado e de um profissional de referência (Costa; Azevedo; Vilar, 2019), a ausência de um sistema de registro eletrônico de saúde para o compartilhamento das informações (Pena; Melleiro, 2018), até desarticulação entre as práticas clínicas desenvolvidas por diferentes profissionais (Noce, 2020). São requeridos estímulos e condições para a execução do cuidado multiprofissional, para tanto, uma das ações necessárias condiz com a inserção de momentos para a discussão crítica acerca da temática desde a formação acadêmica, fortalecendo sua disseminação através da educação permanente, garantindo consequentemente, que o cuidado prestado seja sincronizado, humanizado, com qualidade, resolutivo e seguro.

As limitações deste estudo são próprias de pesquisas com entrevistas, como situações momentâneas e viés de memória dos sujeitos. Adicionalmente, em decorrência do delineamento qualitativo, os resultados obtidos não podem ser extrapolados, o que não diminui sua importância. Nesse sentido, estimulam-se o desenvolvimento de estudos que abordem a pluralidade do tema em outros contextos.

\section{Conclusão}

Ao reproduzir as dúvidas apresentadas por pacientes sobre condutas inerentes aos profissionais de saúde durante o período de internação hospitalar, evidenciou-se que a falha na comunicação foi a grande temática que subsidiou as percepções dos sujeitos envolvidos.

Esse resultado reforça a importância de investimentos tanto em relações interpessoais, habilidades de comunicação e literacia na formação de profissionais da saúde, quanto na discussão e reflexão crítica desses elementos na prática dos serviços de saúde e suas relações sobre o estabelecimento de vínculos entre profissionais e pacientes e sobre o sucesso do tratamento.

Sugere-se que pesquisas futuras sejam desenvolvidas em diversas instituições hospitalares, abrangendo todos os setores existentes. O reconhecimento sobre o potencial da comunicação que acontece nas instituições, especificamente entre profissionais, pacientes e familiares, deve estimular a manutenção e ou treinamentos voltados a equipe multiprofissional atuante, com intuito de potencializar as relações e cuidados em saúde. 


\section{Referências}

Barboza, A., Casavieja, D, López, S., Marina, K., \& Toledo, N. (2020). Opinión de la población migrante sobre los cuidados del equipo de salud en dos policlínicas municipales de Montevideo. Enfermería: Cuidados Humanizados, 9(1), 44-53.

Bardin, L (2016). Análise de conteúdo: edição revista e ampliada. Edições 70.

Brasil a (2013). Ministério da Saúde. Política Nacional de Humanização. http://www.saude.gov.br/humanizasus

Brasil b (2013). Ministério da Saúde. Protocolo de Segurança na Prescrição, uso e Administração de Medicamentos. Agência Nacional de Vigilância Sanitária (ANVISA). https://www.saude.go.gov.br/files//banner_coronavirus/protocolos-assistenciais/11.\%20PROTOCOLO\%20DE\%20SEGURAN\% C3\%87A\% 20N A\% 20 PRESCRI\%C3\%87\%C3\%83O,\%20USO\%20E\%20ADMINISTRA\%C3\%87\%C3\%83O\%20DE\%20MEDICAMENTOS.pdf

Brasil c (2013). Ministério da Saúde. Secretaria de Atenção à Saúde. Departamento de Atenção Especializada. Portaria No 3.390 , De 30 De Dezembro De 2013, Institui a Política Nacional de Atenção Hospitalar (PNHOSP) no âmbito do Sistema Único de Saúde (SUS), estabelecendo- se as diretrizes para a organização do componente hospitalar da Rede de Atenção à Saúde (RAS). http://bvsms.saude.g ov.br/bvs/saudele gis/gm/2013/prt3390_30_12_2013.html

Cancino, K. D., Arias, M., Caballero, E., \& Escudero, E (2020). Development of a safe drug administration assessment instrument for nursing students. Revista Latino-Americana de Enfermagem, Ribeirão Preto, 28 (e3246).

Caprara, A., Franco, A. L.e S (1999). A Relação paciente-médico: para uma humanização da prática médica. Cadernos de Saúde Pública, 15 (3): 647-654.

Coleman, C. A., Peterson, P. S., \& Bumsted, T. (2016). Long-Term Effects of a Health Literacy Curriculum for Medical Students. Famyli Medicine, 48 (1): 49-53.

Costa, M. V. da, Azevedo, G. D., \& Vilar, M. J. P (2019). Aspectos institucionais para a adoção da Educação Interprofissional na formação em enfermagem e medicina. Saúde em Debate, 43(spe1), p: 64-76.

Gil, A. C (2008). Métodos e técnicas de pesquisa social. Atlas, 6.

Grosseman, S., \& Patricio, Z. M (2020). A Relação Médico-Paciente e o Cuidado Humano: Subsídios para Promoção da Educação Médica. Revista Brasileira de educação médica, 28 (2), 99-105.

Koerich, C., Erdmann, A. L., \& Melo, G. M. de M (2020). Interação profissional na gestão da tríade: educação permanente em saúde, segurança do paciente e qualidade. Revista Latino-Americana de Enfermagem, 28 (e3379).

Luiz, F. F., Caregnato, R. C. A., \& Costa, M. R. da (2017). Humanização na Terapia Intensiva: percepção do familiar e do profissional de saúde.Revista Brasileira de Enfermagem, 70(5), 1040-1047.

Luker, J., Murray, C., Lynch, E., Bernhardsson, S., Shannon, M., \& Bernhardt, J (2017). Carers' Experiences, Needs, and Preferences During Inpatient Stroke Rehabilitation: A Systematic Review of Qualitative Studies. Archives of Physical Medicine and Rehabilitation, 98 (9), $1852-1862 . e 13$.

Noce, L. G. de A., Oliveira, T. S. de, Melo, L. C., Silva, K. F. B., Parreira, B. D. M., \& Goulart, B. F (2020). Interprofessional relationships of a patient assistance team in critical care. Revista Brasileira de Enfermagem, 73 (4), 1-7.

Oliveira, R. A. A. de, Zago, M. M. F., \& Thorne, S. E (2017). A interação entre profissionais e sobreviventes do câncer no contexto do cuidado em saúde brasileiro e canadense. Revista Latino-Americana de Enfermagem, 25 (e2972).

Barros, N. F. de, Marin, M. J. S., \& Pinto, A. A. M (2018). Comunicação entre profissional de saúde e paciente: percepções de mulheres com câncer de mama. Nursing, 21 (242): 2272-2276.

Pena, M. M., \& Melleiro, M. M (2018). Eventos adversos decorrentes de falhas de comunicação: reflexões sobre um modelo para transição do cuidado. Revista de Enfermagem da UFSM, 8(3), 616-625.

Sousa, M., Santos, D., Dantas, E., Souza, E., Cavalcanti, L., Araújo, R., et al. (2020). Literácia em saúde e a qualidade de vida da população: revisão integrativa. Revista Eletrônica Acervo Saúde, 51 (e3880), 1-13.

Tavakoly Sany, S., Behzhad, F., \& Ferns, G (2020). Communication skills training for physicians improves health literacy and medical outcomes among patients with hypertension: a randomized controlled trial. BMC Health Services Research, 20 (60): 2-10.

Warrillow, S., Farley, K. J., \& Jones, D (2016). How to improve communication quality with pacients and relatives in the ICU. Minerva Anestesiology, 82 (7), 797-803. 